\title{
Myelin/oligodendrocyte glycoprotein-deficient (MOG-deficient) mice reveal lack of immune tolerance to MOG in wild-type mice
}

\author{
Cécile Delarasse,${ }^{1}$ Philippe Daubas,${ }^{1}$ Lennart T. Mars,${ }^{1}$ Csaba Vizler, ${ }^{1}$ \\ Tobias Litzenburger, ${ }^{2}$ Antonio Iglesias, ${ }^{2}$ Jan Bauer, ${ }^{3}$ Bruno Della Gaspera, ${ }^{1}$ \\ Anna Schubart, ${ }^{2}$ Laurence Decker, ${ }^{1}$ Dalia Dimitri, ${ }^{1}$ Guy Roussel, ${ }^{4}$ Andrée Dierich, ${ }^{5}$ \\ Sandra Amor, ${ }^{6,7}$ André Dautigny, ${ }^{1}$ Roland Liblau, ${ }^{1}$ and Danielle Pham-Dinh ${ }^{1}$
}

\author{
${ }^{1}$ Institut National de la Santé et de la Recherche Médicale (INSERM), Unité 546, Université Pierre et Marie Curie, \\ Hôpital de la Salpêtrière, Paris, France \\ ${ }^{2}$ Max-Planck-Institut für Neurobiologie, Martinsried, Germany \\ ${ }^{3}$ Division of Neuroimmunology, Brain Research Institute, University of Vienna, Vienna, Austria \\ ${ }^{4}$ Centre de Neurochimie, Strasbourg, France \\ ${ }^{5}$ Institut de Génétique et de Biologie Moléculaire et Cellulaire, Centre National de la Recherche Scientifique, \\ INSERM, Université Louis Pasteur, Illkirch, France \\ ${ }^{6}$ Imperial College Faculty of Medicine, Charing Cross Campus, London, United Kingdom \\ ${ }^{7}$ Department of Immunobiology, Biomedical Primate Research Center, Rijswijk, The Netherlands
}

\begin{abstract}
We studied the immunological basis for the very potent encephalitogenicity of myelin/oligodendrocyte glycoprotein (MOG), a minor component of myelin in the CNS that is widely used to induce experimental autoimmune encephalomyelitis (EAE). For this purpose, we generated a mutant mouse lacking a functional mog gene. This MOG-deficient mouse presents no clinical or histological abnormalities, permitting us to directly assess the role of MOG as a target autoantigen in EAE. In contrast to WT mice, which developed severe EAE following immunization with whole myelin, MOG-deficient mice had a mild phenotype, demonstrating that the anti-MOG response is a major pathogenic component of the autoimmune response directed against myelin. Moreover, while MOG transcripts are expressed in lymphoid organs in minute amounts, both MOG-deficient and WT mice show similar $\mathrm{T}$ and $\mathrm{B}$ cell responses against the extracellular domain of MOG, including the immunodominant MOG 35-55 T cell epitope. Furthermore, no differences in the fine specificity of the $T$ cell responses to overlapping peptides covering the complete mouse MOG sequence were observed between $\mathrm{MOG}^{+/+}$and $\mathrm{MOG}^{-/-}$mice. In addition, upon adoptive transfer, MOG-specific $\mathrm{T}$ cells from WT mice and those from MOG-deficient mice are equally pathogenic. This total lack of immune tolerance to MOG in WT C57BL/6 mice may be responsible for the high pathogenicity of the anti-MOG immune response as well as the high susceptibility of most animal strains to MOG-induced EAE.
\end{abstract}

J. Clin. Invest. 112:544-553 (2003). doi:10.1172/JCI200315861.

\section{Introduction}

Experimental autoimmune encephalomyelitis (EAE) is an inflammatory demyelinating disease of the CNS

Received for publication May 3, 2002 and accepted in revised form May 27, 2003.

Address correspondence to: Danielle Pham-Dinh, INSERM U546, Hôpital de la Salpêtrière, 105 Boulevard de l'Hôpital, Paris 75013, France.

Phone: 33-01-40-77-81-45; Fax: 33-01-40-77-81-17;

E-mail: danielle.pham-dinh@chups.jussieu.fr.

André Dautigny, Roland Liblau, and Danielle Pham-Dinh contributed equally to this work.

Conflict of interest: The authors have declared that no conflict of interest exists.

Nonstandard abbreviations used: experimental autoimmune encephalomyelitis (EAE); myelin/oligodendrocyte glycoprotein (MOG); myelin basic protein (MBP); proteolipid protein (PLP); recombinant MOG (rMOG); enzyme-linked immunospot (ELISPOT). that is primarily mediated by $\mathrm{CD} 4^{+} \mathrm{T}$ cells specific for CNS autoantigens (1). Based on similarities in disease susceptibility, course, and histology, EAE is currently used as an animal model for the human disease multiple sclerosis, a common inflammatory and demyelinating disease of the CNS (2).

Tolerance against self-proteins is established and maintained through complex mechanisms in both the thymus and the peripheral lymphoid organs (central and peripheral tolerance, respectively). Positive and negative selection in the thymus is crucial for the development of a self-tolerant $\mathrm{T}$ cell repertoire. Thymocytes that express $\mathrm{T}$ cell receptors with high avidity for autologous peptides are eliminated by programmed cell death or differentiate into an anergic peripheral regulatory subset (3), whereas those that express $\mathrm{T}$ cell receptors with low avidity differentiate into mature $\mathrm{T}$ cells. Potentially autoreactive $\mathrm{T}$ cells nevertheless 
escape thymic deletion and can, upon activation, induce autoimmune diseases. T cell reactivity toward myelin proteins was initially thought to result from selective expression of these antigens beyond the blood-brain barrier. However, recent findings have shown that some isoforms of organ-specific antigens such as myelin basic protein (MBP) and proteolipid protein (PLP) are unexpectedly expressed in the thymus (4-6), a phenomenon called "ectopic" or "promiscuous" gene expression, resulting in profound $\mathrm{T}$ cell tolerance toward these self-antigens (5-8).

Myelin/oligodendrocyte glycoprotein (MOG), a 26to $28-\mathrm{kDa}$ glycoprotein, was first described as a target antigen for the autoimmune demyelination response observed in animals immunized with CNS homogenates $(9,10)$. MOG accounts for about $0.1 \%$ of total CNS myelin protein. The mog gene belongs to the immunoglobulin gene superfamily and is located within the MHC (11). The physiological function of MOG is unknown, but its presence on the outermost lamellae of mature CNS myelin and its late appearance during myelinogenesis suggest that it contributes to myelin maturation or maintenance. MOG differs from other myelin autoantigens because of its immunodominance $(12,13)$ and its highly encephalitogenic properties upon immunization of susceptible animals (14-17). Nevertheless, the relative contribution of MOG as a target antigen with respect to other myelin self-antigens in EAE remains to be defined. In addition, whereas immune tolerance to other myelin antigens has been found to develop both in the thymus and in the periphery, the issue of whether tolerance to MOG develops naturally has not been investigated. To directly address these questions, we generated MOG-null mice and analyzed their clinical and histological severity of EAE, as well as their $\mathrm{T}$ and $\mathrm{B}$ cell responses to MOG, in comparison to those of WT mice.

\section{Methods}

Generation of MOG knockout mice. Genomic DNA fragments of $m o g$, isolated from a recombinant $\lambda$ phage (18), were subcloned into the pBluescript KS vector (Stratagene, La Jolla, California, USA). The $n l a c Z$ sequences were inserted into a vector containing a 4-kb fragment of $5^{\prime} \operatorname{mog}$ sequences at the unique BstEII site in exon 1 , seven nucleotides upstream of the initiation codon. A neoR gene under the control of the HSVtk promoter and a $6-\mathrm{kb}$ fragment of $3^{\prime}$ mog sequences were then subcloned in the BstEII site. The final targeting vector was linearized at the unique HindIII site in the pKS polylinker and used for electroporation.

Growth, transfection, and selection of D3 ES cells (originating from 129S2/SVPas mice, $\mathrm{H}-2^{\mathrm{b} / \mathrm{b}}$ ) and the generation of chimeric mice were performed as previously described (19). One positive clone was identified by Southern blot analysis and injected into C57BL/6 blastocysts.
$D N A$ and RNA analyses. Probes for Southern blot analyses were labeled by random priming of the 1.25$\mathrm{kb}$ SacI-HindIII DNA fragment. Mouse genotypes were determined by PCR amplification of tail DNA. The WT MOG allele was characterized by amplification of a 162-bp fragment with a sense primer in exon 1 ( $5^{\prime}$-AGGAAGGGACATGCAGCCGGAG-3') and an antisense primer in exon 1 ( $5^{\prime}$-CTGCATAGCTGCATGACAACTG-3'). A 470-bp fragment of mutated MOG was amplified with the same sense primer, and an antisense primer in the nlac $Z$ sequence ( $5^{\prime}$-GATGGGCGCATCGTAACCGTGC-3').

For Northern blot analyses, RNA isolated from tissues with the RNeasy Midi Kit (QIAGEN Inc., Valencia, California, USA) was denatured with glyoxal and DMSO, run on a $1 \%$ agarose gel, and blotted onto Hybond $\mathrm{N}^{+}$positively charged nylon membrane (Amersham Biosciences Corp., Piscataway, New Jersey, USA). The blot was probed with the ${ }^{32} \mathrm{P}$-labeled random-primed mouse MOG CDNA, and exposed with an intensifying screen at $-80^{\circ} \mathrm{C}$. RT-PCR analysis of MOG expression in lymphoid organs was performed using one whole thymus and one whole spleen for total RNA preparations. Total RNA was reverse transcribed into cDNA using random hexaprimers (Promega Corp., Madison, Wisconsin, USA) and Omniscript Reverse Transcriptase kit (QIAGEN Inc.).

PCR analysis. PCR amplification was performed using the Q-Solution (QIAGEN Inc.). The amplified sequences were normalized with respect to $\beta$-actin expression. PCR conditions comprised a single denaturing step at $94^{\circ} \mathrm{C}$ for 5 minutes, 30 cycles (to amplify actin cDNA) or 40 cycles (for PLP and MOG cDNA) at $95^{\circ} \mathrm{C}$ for 1 minute, $64^{\circ} \mathrm{C}$ (actin and PLP) or $66^{\circ} \mathrm{C}$ (MOG) for 1 minute, and $72^{\circ} \mathrm{C}$ for 3 minutes, and a final elongation step at $72^{\circ} \mathrm{C}$ for 5 minutes. PLP/ DM20 primers were $5^{\prime}$-TTGGAGTCAGAGTGCCAAAGAC-3' and 5'-AGCAATAAACAGGTGGAAGGTC-3'; MOG primers were $5^{\prime}$-GACCTCAGCTTGGCCTGACCC- $3^{\prime}$ and $5^{\prime}$-TGCTGGGCTCTCCTTCCGC-3'; and $\beta$-actin primers were $5^{\prime}$-GGTTCCGATGCCCTGAGGCTC- $3^{\prime}$ and $5^{\prime}$-ACTTGCGGTGCACGATGGAGG-3'.

Western blot analysis. Total protein extracts $(100 \mu \mathrm{g})$ of mouse brain were run on an SDS polyacrylamide gel, then electroblotted onto a nitrocellulose membrane (Amersham Biosciences Corp.). The membrane was incubated with anti-MOG antibodies directed against the carboxy-terminal or the Ig-like extracellular domain of MOG (1:1,000 dilution) (20), then with secondary peroxidase-conjugated goat anti-rabbit IgG (Sigma-Aldrich, St. Louis, Missouri, USA) and then processed according to ECL protocol (Amersham Biosciences Corp.).

Histology, immunohistochemistry, and electron microscopy. $\beta$-Gal activity was revealed as previously described (19) in mice perfused through the heart with cold $4 \%$ paraformaldehyde.

MOG protein was detected by immunohistochemistry on frontal cryostat brain sections. The lac $Z$ gene 
product was detected simultaneously with a mouse anti- $\beta$-gal $\mathrm{mAb}$ (Sigma-Aldrich) added to the incubation medium. MOG and $\beta$-gal-specific antibodies were revealed with fluorescein-conjugated goat antirabbit IgG and TRITC-labeled goat anti-mouse IgG antibodies, respectively.

The brain and spinal cord of EAE mice were removed and embedded in paraffin. The extent of inflammation and demyelination was evaluated on $3-\mu \mathrm{m}$ spinal cord cross sections stained with $\mathrm{H} \& \mathrm{E}$, anti-CD3 mAb, and Klüver-Barrera myelin stain. The inflammatory index represents the average number of inflammatory infiltrates per spinal cord section, with $10-15$ sections studied per mouse.

For electron microscopy, ultrathin sections were stained with lead citrate and observed with an EM420 Philips electron microscope. The negatives were scanned, and the myelin sheaths were analyzed quantitatively with Image Tools software.

Induction of active and passive EAE. Mice were housed under conventional conditions. Eight- to 12-week-old littermates, backcrossed for eight generations on a C57BL/ 6 background $\left(\mathrm{H}-2^{\mathrm{b} / \mathrm{b}}\right)$, were included in EAE and immunological experiments.

For active EAE, $\mathrm{MOG}^{+/+}$and $\mathrm{MOG}^{-/-}$littermates were immunized subcutaneously with $200 \mu \mathrm{g}$ of rat recombinant MOG (rat rMOG, amino acids 1-125) (12), or $1.3 \mathrm{mg}$ of mouse CNS myelin purified on a sucrose density gradient associated with osmotic shock steps (21), in CFA containing $600 \mu \mathrm{g}$ of Mycobacterium tuberculosis H37RA (Difco Laboratories, Detroit, Michigan, USA) according to a previously described protocol (22). For EAE induction, animals received additional intravenous injections of pertussis toxin (List Biological Laboratories Inc., Campbell, California, USA) on day 0 (200 ng) and day 2 (400 ng).

For passive EAE, age- and sex-matched WT or $\mathrm{MOG}^{-/-} \mathrm{C} 57 \mathrm{BL} / 6$ mice were immunized in the hindleg footpads with $100 \mu \mathrm{g}$ mouse rMOG (amino acids 1-116) emulsified in CFA. Ten days later, the draining lymph nodes were collected and single-cell suspensions were cultured for 72 hours at $5 \times 10^{6}$ cells $/ \mathrm{ml}$ in serum-free medium (HL-1; BioWhittaker Inc., Walkersville, Maryland, USA) in the presence of mouse rMOG $(10 \mu \mathrm{g} / \mathrm{ml})$ and IL-12 $(5 \mathrm{ng} / \mathrm{ml})$. Cells were washed and diluted at $30 \times 10^{6}$ in $0.2 \mathrm{ml}$ PBS before being injected intravenously into naive WT recipients (23). The adoptive transfer of resting $T$ cells was performed as described before (24). Purified $\mathrm{T}$ cells $\left(40 \times 10^{6}\right.$ cells) were injected intravenously into syngeneic $\mathrm{T}$ cell receptor $\mathrm{C}^{-/-}$recipients (a kind gift of O. Lantz, INSERM U520, Paris, France) 48 hours before subcutaneous immunization with 200 $\mu \mathrm{g}$ rat $\mathrm{rMOG}$ in CFA at the base of the tail and a single intravenous injection of pertussis toxin $(200 \mathrm{ng})$.

The mice were scored daily for clinical signs of EAE as previously described (12). Mean maximal EAE severity was compared using the two-tailed
Student $t$ test, mortality was compared using the Fisher's exact test, and delay in disease onset was assessed using the log-rank test.

$T$ cell proliferation assays. Age- and sex-matched mice were immunized in the footpads of the hind legs with $100 \mu \mathrm{g}$ mouse rMOG, or a pool of overlapping 15 -mer peptides $(200 \mu \mathrm{g} / \mathrm{ml}$ each $)$ spanning the whole mouse MOG sequence (12) emulsified in CFA. Ten days later, the popliteal and inguinal lymph node cells were cultured for 72 hours in triplicate at a concentration of $4.5 \times 10^{5}$ cells per well in flat-bottomed 96-well plates in serum-free medium (HL-1; BioWhittaker Inc.) in the presence or absence of antigens. Proliferation was measured by incorporation of ${ }^{3} \mathrm{H}$-thymidine (Amersham Biosciences Corp.) during the last 24 hours of culture at $1 \mu \mathrm{Ci} /$ well. Only animals with comparable control responses to the purified protein derivative of $M$. tuberculosis H37RA were included in the analyses.

ELISA for serum anti-MOG IgG levels. Serum was obtained after coagulation and centrifugation of peripheral blood from mice before and after footpad immunization with rat rMOG emulsified in CFA. Anti-rat rMOG and anti-MOG peptides IgG were measured by ELISA at an OD of $405 \mathrm{~nm}$, on serum

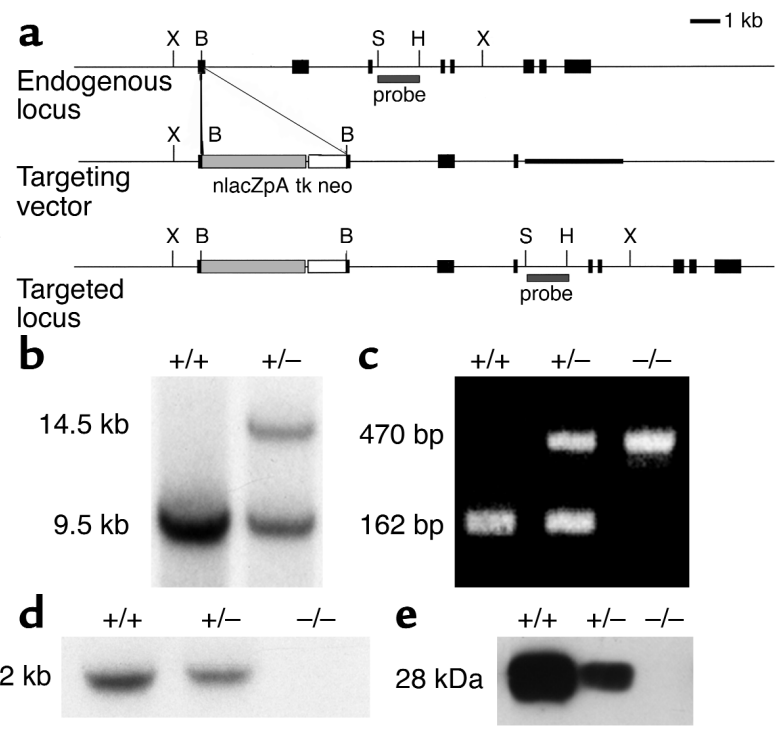

Figure 1

Targeted disruption of the mouse MOG locus. (a) Partial restriction maps of the WT MOG allele (endogenous locus), the targeting vector, and the expected targeted locus. The location of the external screening probe is shown. X, Xmnl; B, BstEll; S, Sacl; H, HindIII. (b) Southern blot analysis of DNA from the parental ES cell line $(+/+)$ and the targeted clone $(+/-)$, digested with $X m n l$ and hybridized with the external screening probe. This probe detects a WT 9.5-kb fragment or a $14.5-\mathrm{kb}$ recombinant fragment. (c) Ethidium bromide-stained PCR products from tail DNA. The 470-bp and 162-bp fragments correspond to the mutated and the WT alleles, respectively. (d) Northern blot analysis of RNA isolated from the brain of $\mathrm{MOG}^{+/+}, \mathrm{MOG}^{+/-}$, and $\mathrm{MOG}^{-/-}$mice probed for MOG transcripts. (e) Western blot of brain proteins from 1-month-old $\mathrm{MOG}^{+/+}, \mathrm{MOG}^{+/-}$, and $\mathrm{MOG}^{-/-}$ mice probed with anti-MOG antibodies. 
diluted $1: 60,1: 360$, and $1: 2,160$, as previously described (25), by an investigator unaware of the genotype of the mice. Background OD values on wells coated solely with BSA (blocking buffer) ranged from 0.175 to 0.220 (mean 0.202 ).

IFN- $\gamma$ enzyme-linked immunospot. Fourteen days after immunization with MOG 35-55 peptide, IFN- $\gamma$ releasing unfractionated or $\mathrm{CD}^{+}-$ depleted splenocytes were quantified by cytokine-specific enzyme-linked immunospot (ELISPOT) analysis as described previously (26). Depletion of $\mathrm{CD}^{+} \mathrm{T}$ cells $(>99.5 \%)$ from mouse splenocytes was achieved by magnetic cell-sorting (MACS) (Miltenyi Biotech Inc., Auburn, California, USA). Individual, unfractionated, or CD8-depleted spleen cultures were incubated in vitro for 40 hours on IFN- $\gamma$ ELISPOT plates in quadruplicate in the presence or absence of antigen $(50 \mu \mathrm{g} / \mathrm{ml})$. The percentage of $\mathrm{CD}^{+} \mathrm{T}$ cells was determined by FACS analysis of the preculture splenocyte populations using an anti-mouse CD3 $\varepsilon$ mAb (Pharmingen, San Diego, California, USA). The CD3 mAb response was saturating in each mouse irrespective of the genotype.

\section{Results}

Generation and characterization of MOG-null mice. The gene encoding mouse MOG (18) was disrupted in ES cells by homologous recombination using a cassette encoding $\beta$-gal with a nuclear localization sequence (nlacZ) inserted into exon 1 of the mog gene (Figure 1, a-c). The inactivation of the mog gene was assessed by Northern blot and Western blot analyses, which demonstrated that MOG transcripts (Figure 1d) and MOG protein (Figure 1e) were indeed absent in $\mathrm{MOG}^{-1-}$ mutant brains and were reduced in $\mathrm{MOG}^{+/-}$ animals compared with WT controls. $\mathrm{MOG}^{-/}$animals bred well, had no physical or behavioral abnormalities, and appeared phenotypically indistinguishable from WT or heterozygous $\mathrm{MOG}^{+/-}$mice up to 20 months of age.

$\beta$-Gal activity detected histochemically in adult $\mathrm{MOG}^{+/-}$animals was restricted to oligodendrocytes and corresponded well with

\section{Figure 2}

the known topography of MOG expression (11) (Figure 2a). The reaction product was first detected in the CNS at postnatal day 10 in the brain stem and the ventral spinal cord. Later in postnatal

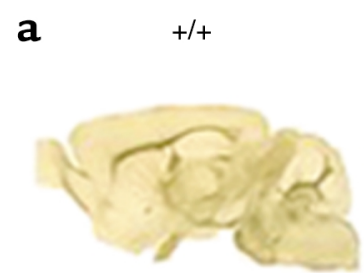

b
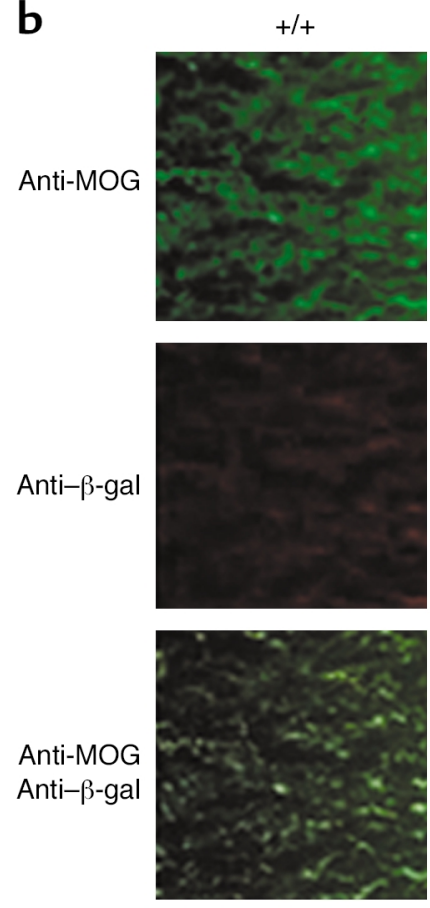

C

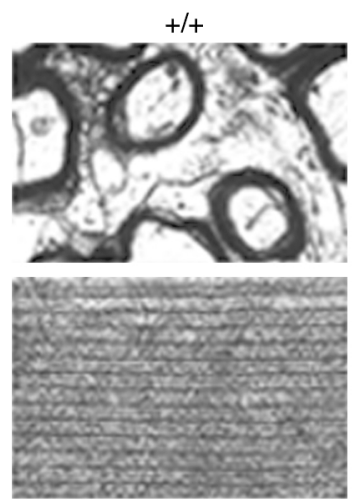

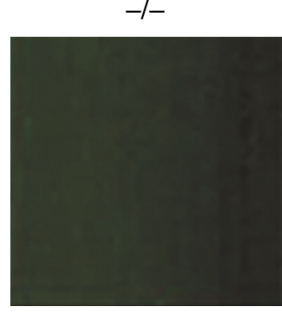
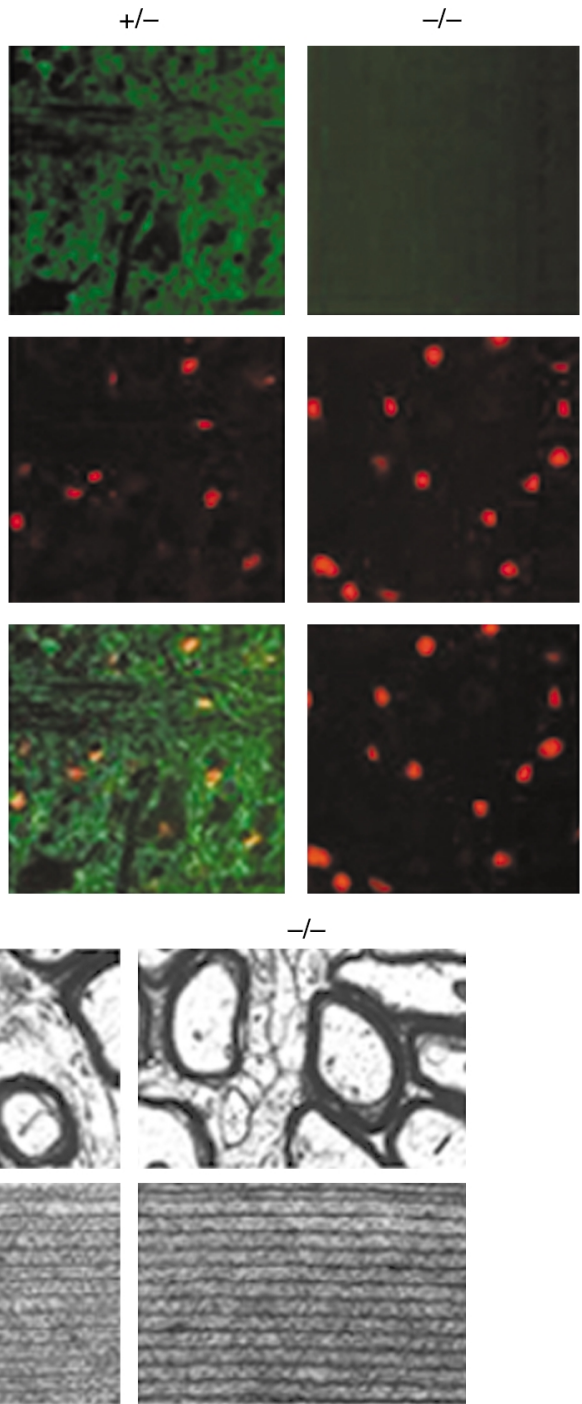

Histological analyses of brains from $\mathrm{MOG}^{+/+}$, nlacZ-MOG ${ }^{+/-}$, and nlacZ-MOG ${ }^{-/}$animals. (a) Histochemical detection of $\beta$-gal activity on brain sections using $X$-gal as substrate. Left panel: No reaction product is observed in the brain of a $\mathrm{MOG}^{+/+}$mouse. Middle panel: X-gal staining of an nlacZ-MOG ${ }^{+/}$mouse. Blue reaction product is located in brain areas where mog is known to be transcribed. Right panel: Enlargement of a region (indicated by the rectangle) of the corpus callosum of the nlacZ- $\mathrm{MOG}^{+/-}$mouse. (b) Immunohistofluorescent detection of $\mathrm{MOG}$ and $\beta$-gal in brain stem of $\mathrm{MOG}^{+/+}$, nlacZ-MOG ${ }^{+/}$, or nlacZ-MOG-1- mice with MOG-specific (green) and $\beta$-gal-specific (red) antibodies. (c) Electron micrographs of striatum sections of $\mathrm{MOG}^{+/+}$and $\mathrm{MOG}^{-/-}$ mice. The myelin sheaths in both types of mice show normal compaction, characterized by major dense and intraperiodic lines. Top, $\times 28,100$; bottom, $\times 260,600$. 


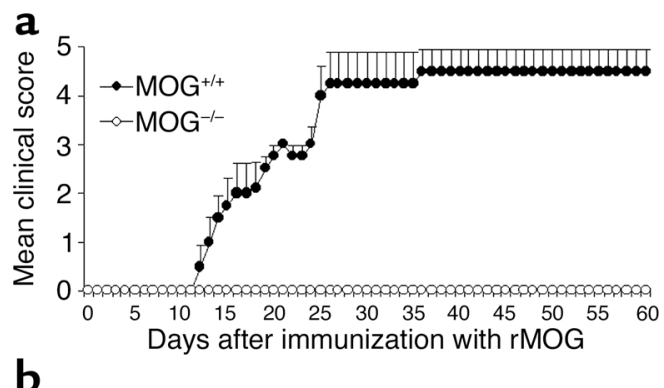

\section{c}
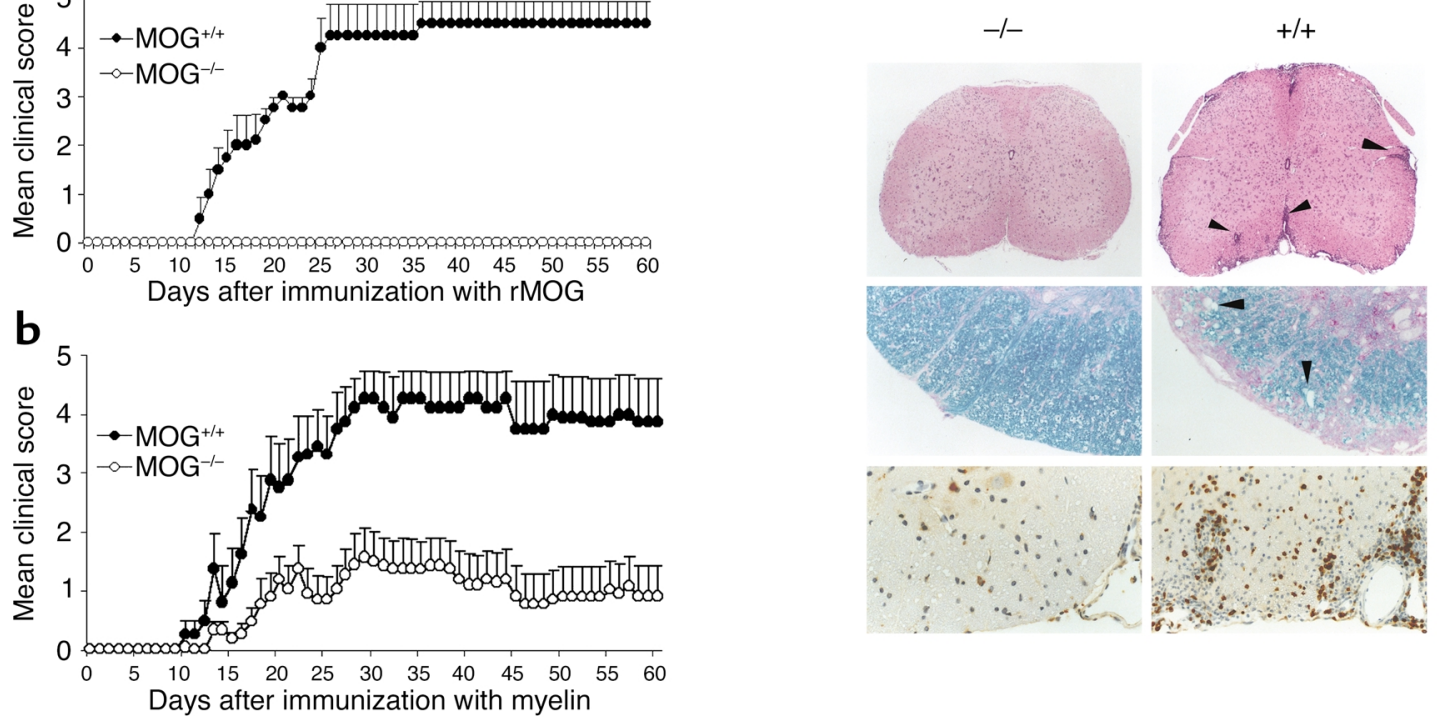

\section{Figure 3}

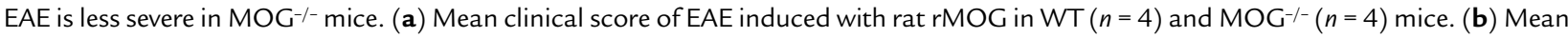
clinical score of two independent EAE experiments induced with whole myelin in WT $(n=8)$ and $\mathrm{MOG}^{-/-}(n=13)$ female mice. $\mathrm{MOG}^{+/+}$

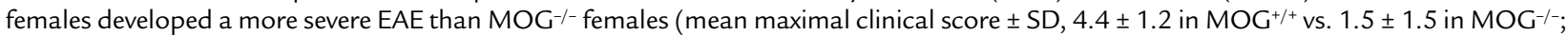
$P=0.004$, two-tailed Student $t$ test), leading to higher mortality ( $75 \%$ in $\mathrm{MOG}^{+/+}$vs. $15 \%$ in $\mathrm{MOG}^{-/-} ; P=0.006$, Fisher's exact test). Disease onset was also delayed in $\mathrm{MOG}^{-1-}$ mice (mean $\pm \mathrm{SD}, 17.9 \pm 6.6$ days) as compared with WT mice (13.4 days $\pm 1.9 ; P=0.008$, log-rank test). (c) CNS histology of $\mathrm{MOG}^{-1-}$ (left column) and WT mice (right column) at day 20 after induction of EAE with whole myelin. Upper panels ( $\times 11$ ): H\&E staining showing large infiltrates (arrowheads) in WT mice but no inflammation in $\mathrm{MOG}^{-/-}$mice. Middle panel ( $\left.\times 70\right)$ : KlüverBarrera staining for myelin shows no loss of myelin in $\mathrm{MOG}^{-/-}$mice, but loss of subpial myelin in WT mice. Arrowheads indicate the presence of intramyelinic vacuoles. Lower panel $(\times 70)$ : Immunocytochemistry for CD3 shows the absence of T cell inflammation in MOG-1mice, whereas WT mice have large numbers of T cells in inflammatory infiltrates.

development, the strongest staining was found in highly myelinated brain areas such as the corpus callosum, fimbria, and cerebellar white matter. In double-labeled immunofluorescence experiments performed on brain sections of $\mathrm{MOG}^{+/-}$animals, $\beta$-gal immunoreactivity was found only in nuclei of cells with MOG-immunoreactive plasma membranes (Figure $2 \mathrm{~b}$ ). The architecture of myelin sheaths in MOG-null mice was examined by electron microscopy in the corpus callosum, striatum, cerebellum, and optic nerve of 2-, 4-, and 14-month-old animals. There were no differences between $\mathrm{MOG}^{-/-}$and WT mice in myelin sheath structure (Figure 2c), distance between dense lines, distance between axolemma and internal membrane mesaxon, or the ratio of myelin thickness to axon diameter (data not shown). Further, no alterations in the kinetics of myelination were observed. Moreover, by contrast to several other myelin mutants (27), the MOG-deficient mice had no structural abnormalities in axons as assessed by electron microscopy, even in 18month-old mice (data not shown).

$E A E$ is decreased in $M O G^{-/-} C 57 B L / 6$ mice. Due to the absence of MOG expression in MOG-null mice, the MOG-induced immune response should not induce $\mathrm{CNS}$ tissue damage. Indeed, when WT and $\mathrm{MOG}^{-/-}$ mice, backcrossed eight times onto C57BL/6 $\left(\mathrm{H}-2^{\mathrm{b} / \mathrm{b}}\right)$, were immunized with rMOG, or with the MOG
35-55 peptide sequence, all $\mathrm{MOG}^{+/+}$mice developed severe EAE, whereas $\mathrm{MOG}^{-/-}$animals exhibited no clinical (Figure 3a) or histological abnormalities (data not shown).

To investigate the contribution of the anti-MOG immune response to the development of EAE, we induced EAE in $\mathrm{MOG}^{+/+}$and $\mathrm{MOG}^{-/-}$littermates using whole myelin as the immunogen. Whereas WT females $(n=8)$ rapidly developed symmetrical motor deficits starting at day 10-16 after immunization, the disease in $\mathrm{MOG}^{-/}$females $(n=13)$ had a delayed onset $(P=0.008)$ and the clinical signs were significantly milder $(P=0.004)$ (Figure $3 b)$. Similar results were obtained in two independent experiments involving $\mathrm{MOG}^{+/+}(n=11)$ and $\mathrm{MOG}^{-/-}$ $(n=13)$ males, although the overall severity of disease was less than in females (data not shown). Histological analyses of the CNS were performed in additional groups of mice at day 20-24 after immunization, during the progressive phase of EAE (mean clinical score 2.2 in $\mathrm{MOG}^{+/+}$mice, $n=4$; and 0.5 in $\mathrm{MOG}^{-/-}$littermates, $n=8$ ). $\mathrm{MOG}^{+/+}$mice showed clear signs of inflammation and some mild demyelination. In $\mathrm{MOG}^{-/-}$mice, however, there was little inflammation, and never demyelination (Figure 3c). At day 60 after immunization, meningeal inflammation was still more pronounced in $\mathrm{MOG}^{+/+}$mice (inflammatory index $2.3 \pm 1.0, n=4$ ) 
than in $\mathrm{MOG}^{-/-}$mice (inflammatory index $0.9 \pm 0.5$, $n=5)$, in which, even at this late time point, no demyelination was observed.

In WT mice, the weak MOG transcript expression in lymphoid organs correlates with a lack of immune tolerance to MOG. Expression in the thymus and peripheral hematopoietic organs may result in immunological tolerance or influence the function of MOG-specific $T$ cell repertoire, as demonstrated recently for the major myelin proteins $\operatorname{MBP}(4,28)$ and PLP $(5,6)$. Hence we examined whether the potent immunogenicity of MOG was associated with a lack of MOG expression in hematopoietic tissues. RT-PCR analysis of thymus, spleen, liver, and brain (as a positive control) from 3 -month-old WT C57BL/ 6 mice was performed with MOG- and PLPspecific primers. As expected, both PLP and its alternative transcript DM20 were detected in brain, whereas, as recently reported $(5,6)$, DM20 was by far the predominant PLP gene transcript expressed in lymphoid organs (Figure 4). Similarly to full-length PLP transcripts, the MOG transcripts were only detected in minute amounts in both thymus and spleen and were absent in the liver (Figure 4).

In view of the high pathogenicity of the anti-MOG immune response and the low level of MOG transcripts in lymphoid organs, we assessed whether immune tolerance to MOG develops in $\mathrm{MOG}^{+/+}$ mice. To this end, we compared $\mathrm{T}$ and $\mathrm{B}$ cell responses to $\mathrm{MOG}$ in $\mathrm{MOG}^{+/+}$versus $\mathrm{MOG}^{-/-}$mice after conventional immunization with rMOG or MOG peptides. In MOG-deficient mice, no tolerance to MOG should develop, and, as a result, the immune response to MOG following immunization represents the uncensored maximal response. Secondary in vitro $\mathrm{T}$ cell-proliferative responses to rMOG (Figure 5b), as well as to the 31 overlapping mouse MOG peptides (Figure 5a), were identical in both genotypes. Incidentally, this analysis revealed the immunogenicity in C57BL/ 6 mice of three peptides localized in the transmembrane or in the second hydrophobic region of the MOG protein.

Similarly, IFN- $\gamma$ secretion in response to $\mathrm{rMOG}$ was identical in $\mathrm{MOG}^{+/+}$and $\mathrm{MOG}^{-/-}$mice; no IL-4 was detected in any of the groups (data not shown). The presence of an uncensored $\mathrm{T}$ cell response in $\mathrm{MOG}^{+/+}$mice was further corroborated by the use of a sensitive method to enumerate the number of specific IFN- $\gamma$-producing T cells. This ELISPOT approach revealed no significant difference between $\mathrm{MOG}^{+/+}$ and $\mathrm{MOG}^{-/-}$mice in the frequency of $\mathrm{CD}^{+} \mathrm{T}$ cells responding to the dominant MOG 35-55 encephalitogenic peptide (Figure 5c).

The state of tolerance to MOG in the B cell compartment was also compared in both genotypes by measurement of the magnitude of the antibody response at different time points after immunization. Here again, both groups of mice responded similarly (Figure $5 \mathrm{~d}$ ). In addition, when Th1-driven (IgG2a) and
Th2-driven (IgG1) anti-MOG IgG isotypes were specifically measured, no differences were found (data not shown). Moreover, sera from MOG-immunized $\mathrm{MOG}^{-/-}$and $\mathrm{MOG}^{+/+}$littermates recognized the same linear MOG epitopes as detected by ELISA on a panel of 13 overlapping peptides covering the rMOG Ig-like domain sequence (Figure 5e).

Equal encephalitogenic potential of MOG-specific T cells from $\mathrm{MOG}^{+/+}$and $\mathrm{MOG}^{-/-}$mice. To compare the encephalitogenicity of rMOG-specific $\mathrm{T}$ cell responses from $\mathrm{MOG}^{+/+}$and $\mathrm{MOG}^{-/-}$mice in $\mathrm{MOG}^{+/+}$ mice two complementary approaches were investigated. The first was a classical adoptive transfer experiment in which primed rMOG-specific $\mathrm{T}$ cells were transferred into syngeneic recipients following in vitro restimulation (Figure 6a). The second was the transfer of the full resting $\mathrm{T}$ cell repertoire derived from $\mathrm{MOG}^{+/+}$and $\mathrm{MOG}^{-/-}$mice into syngeneic $\alpha \beta$ T cell-deficient recipients, followed by immunization with rMOG (Figure 6b). Although the two protocols induced a distinct course of disease, the onset, intensity, and evolution of EAE induced by $\mathrm{T}$ cells derived from either $\mathrm{MOG}^{+/+}$or $\mathrm{MOG}^{-/}$mice were indistinguishable, irrespective of whether preactivated MOG-specific T cells or undifferentiated resting $T$ cells were transferred.

In summary, in vitro analyses revealed that both the cellular and the humoral immune responses to MOG are quantitatively and qualitatively similar in $\mathrm{MOG}^{+/+}$ and $\mathrm{MOG}^{-/-}$mice. $\mathrm{MOG}^{+/+}$and $\mathrm{MOG}^{-/-}$mice respond similarly to $r M O G$ and to its immunogenic peptides. Moreover, the encephalitogenic potential of both naive and primed MOG-specific T cells from both types of mice is identical. Collectively, these data imply that WT $\mathrm{MOG}^{++}$animals do not develop detectable tolerance to this myelin protein.

\section{Discussion}

To our knowledge this is the first description of MOG-deficient mice. We have chosen a knock-in strategy using an $n l a c Z$ reporter gene to generate a

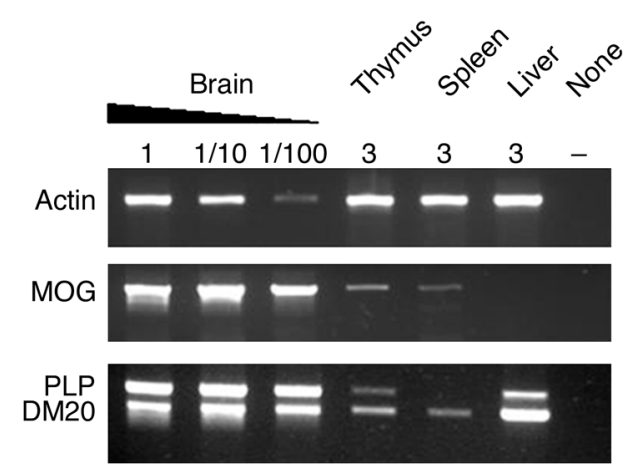

\section{Figure 4}

Low expression of MOG transcripts in lymphoid organs. Expression of PLP and MOG in lymphoid organs, liver, and brain. RNA was prepared from 3-month-old C57BL/ 6 mice. Numbers above lanes indicate microliters of $C D N A$ used in PCR. 

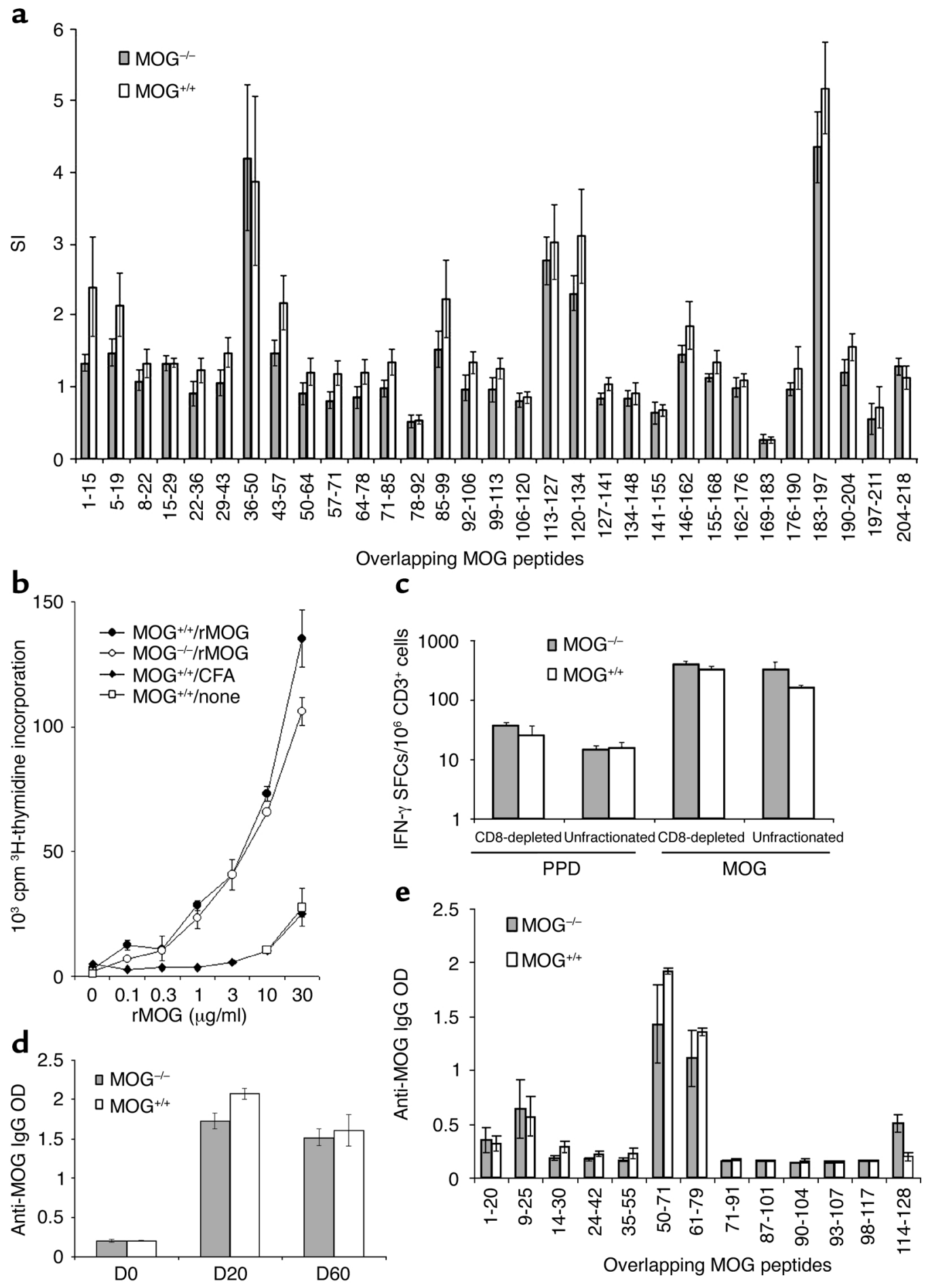

\section{Figure 5}

(a) Proliferative response of lymph node cells $\mathrm{MOG}^{+/+}(n=6)$ and $\mathrm{MOG}^{-/-}(n=6)$ mice 10 days after immunization with 31 overlapping mouse MOG peptides. The in vitro recall response was induced using individual MOG peptides $(200 \mu \mathrm{g} / \mathrm{ml})$. SI, stimulation index. (b) The proliferative response of lymph node cells of MOG ${ }^{+/+}(n=3)$ and $\mathrm{MOG}^{-/-}(n=3)$ mice 10 days after immunization with mouse $\mathrm{rMOG}$. The recall response was elicited with different doses of rMOG. (c) The MOG 35-55-specific T cell population was enumerated by IFN- $\gamma$ ELISPOT in splenocyte cultures of individual $\mathrm{MOG}^{+/+}(n=4)$ and $\mathrm{MOG}^{-/-}(n=3)$ mice 14 days after MOG 35-55 immunization. Unfractionated or CD8-depleted spleen cultures were stimulated with purified protein derivative (PPD) (50 $\mu \mathrm{g} / \mathrm{ml})$, or MOG 35-55 (50 $\mu \mathrm{g} / \mathrm{ml})$. (d) MOG-specific antibody response in $\mathrm{MOG}^{+/+}(n=4)$ and $\mathrm{MOG}^{-/-}(n=4)$ mice at days 0,20 , and 60 after immunization with rat $\mathrm{rMOG}$. MOG-specific IgG levels were assessed by ELISA on serum diluted 1:60; quantitatively similar results were obtained with 1:360 and 1:2,160 dilutions (data not shown). (e) Analysis of the fine specificity of anti-MOG IgG in $\mathrm{MOG}^{+/+}$and $\mathrm{MOG}^{-/-}$mice 40 days after immunization with rat $r$ MOG. Peptide-specific IgGs were assessed by ELISA with a panel of 13 overlapping rat MOG peptides. Comparable results were obtained at days 20 and 60 after immunization. 

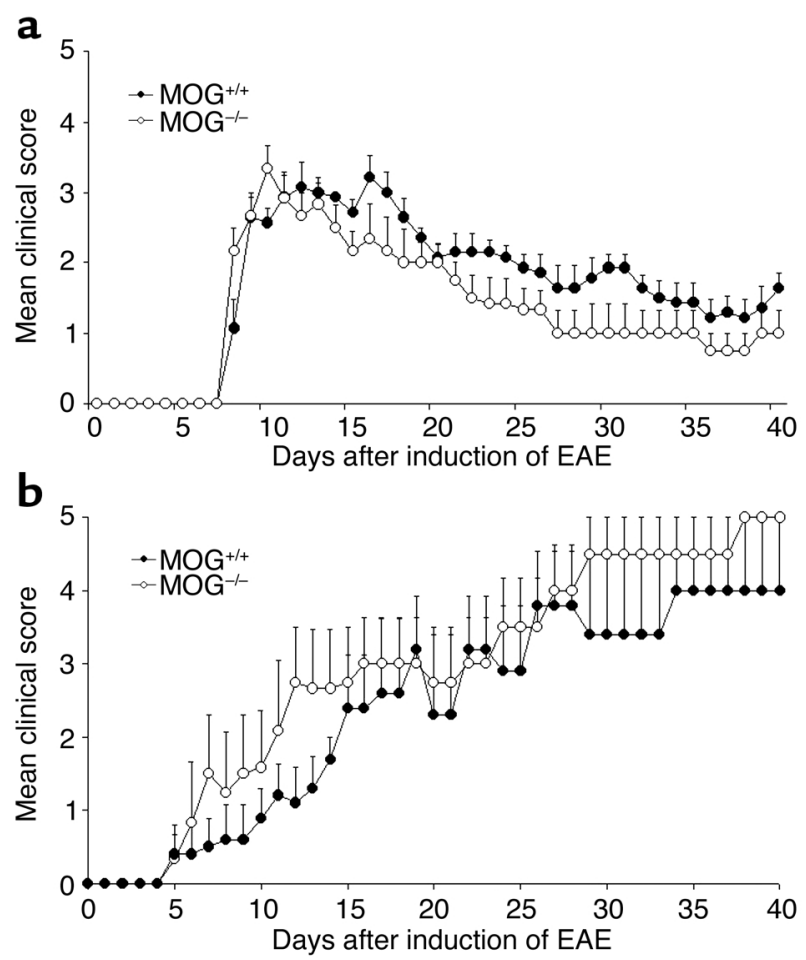

Figure 6

MOG-specific $T$ cells from $\mathrm{MOG}^{-/-}$and $\mathrm{MOG}^{+/+}$mice are equally encephalitogenic. (a) EAE induced by the adoptive transfer of activated MOG-specific T cells from either $\mathrm{MOG}^{-/-}(n=6)$ or $\mathrm{MOG}^{+/+}$ $(n=7)$ mice into WT C57BL/ 6 mice. (b) EAE induced by the adoptive transfer of purified T cells from either $\mathrm{MOG}^{-/-}(n=6)$ or $\mathrm{MOG}^{+/+}(n=5)$ mice into syngeneic $\alpha \beta$ T cell-deficient recipients followed, 48 hours later, by rMOG immunization. For each proto$\mathrm{col}$, data from two experiments are pooled and represent mean clinical score \pm SEM plotted against time.

mouse line in which activation and transcription of the disrupted mog gene can be traced. The homozygous $\mathrm{MOG}^{-/-}$mice appeared indistinguishable from their WT littermates. The lack of clinical, histological, and ultrastructural abnormalities in MOG knockout mice provided us with a unique opportunity to study the contribution of the anti-MOG immune response to the clinical and histological phenotype of EAE. We immunized mice with a whole-myelin preparation containing the full spectrum of myelin antigens. Under the experimental conditions used, the anti-MOG immune response contributed very significantly to the severity of myelin-induced EAE in the $\mathrm{MOG}^{+/+}$mice, as shown by the significant delay in onset and the reduced severity of EAE in $\mathrm{MOG}^{-/}$mice. The non-demyelinating low-grade EAE exhibited by $\mathrm{MOG}^{-/}$ C57BL/6 mice following immunization with whole myelin most likely resulted from an immune response to other encephalitogenic self-antigens present in the myelin preparation. In that respect, native PLP (29), or the PLP 178-191 peptide (30), and the oligodendrocyte-specific protein (31) have been shown to be encephalitogenic in C57BL/6 mice. Other as-yet unknown encephalitogenic components may also contribute. Histologically, ablation of MOG reduced CNS inflammation and prevented demyelination during the exacerbating phase of myelin-induced EAE. It is possible that, in $\mathrm{MOG}^{-/-}$mice, MOG-reactive T cells do not accumulate in the CNS because of the absence of their specific antigen, as previously demonstrated in other systems (32). These differences in EAE severity, both clinical and histological, are therefore very likely a result of the absence of MOG as a target for the anti-MOG immune response. The complete resistance to $\mathrm{EAE}$ of $\mathrm{MOG}^{-/}$mice immunized with rMOG and the similar encephalitogenic potential of $\mathrm{MOG}^{+/+} \mathrm{T}$ cells and $\mathrm{MOG}^{-/-} \mathrm{T}$ cells when transferred to $\mathrm{MOG}^{+/+}$mice strengthen this hypothesis.

Although the encephalitogenic property of MOG in C57BL/6 mice is well established, the important contribution of this minor myelin component to disease severity after immunization with whole myelin was unexpected. We asked, therefore, whether the strong pathogenicity of MOG in WT mice could be due to a lack of immunological tolerance toward this self-antigen. We assessed MOG expression in lymphoid organs because it was recently shown that the expression of several MBP isoforms and the DM20 isoform of PLP in the thymus and in secondary lymphoid organs leads to a strong tolerance toward these major myelin components $(4-6,28)$. The expression of MOG, as well as the full-length isoform of PLP, was barely detectable in the lymphoid organs tested. This low expression level might be insufficient to induce tolerance toward these self-antigens. Indeed, in the thymus of mice, PLP expression is very weak compared with DM20 expression. This correlates with a lack of tolerance to amino acids PLP 139-151, encoded by an exon selectively spliced out in the DM20 transcript, and explains PLP 139-151 peptide's immunogenic and encephalitogenic properties in SJL/J mice, which express MHC class II molecules capable of binding and presenting this peptide to $\mathrm{CD} 4^{+} \mathrm{T}$ cells $(5,6)$.

To evaluate whether the $\mathrm{T}$ and $\mathrm{B}$ cell anti-MOG repertoires in WT $\mathrm{MOG}^{+/+}$mice were censored in any detectable way, we immunized $\mathrm{MOG}^{+/+}$and $\mathrm{MOG}^{-/}$mice with $\mathrm{rMOG}$ and compared the magnitude of both $\mathrm{T}$ and $\mathrm{B}$ cell responses. T cell reactivity to rMOG or an overlapping set of peptides spanning the entire MOG protein, as well as the kinetics and intensity of the total IgG anti-MOG response, were similar in $\mathrm{MOG}^{+/+}$and $\mathrm{MOG}^{-/-}$animals. Likewise, the cellular immune response to the encephalitogenic MOG 35-55 peptide was identical in both groups. Indeed, the size of the responding unfractionated or $\mathrm{CD}^{+} \mathrm{T}$ cell population, as assessed by IFN- $\gamma$ ELISPOT, was indistinguishable between $\mathrm{MOG}^{+/+}$and $\mathrm{MOG}^{-/-}$mice. Moreover, our analysis revealed new immunogenic peptides localized in 
both hydrophobic regions of MOG (20). Strikingly, both $\mathrm{MOG}^{+/+}$and $\mathrm{MOG}^{-/-}$mice mount a similar $\mathrm{T}$ cell in vitro response to these peptides, indicating that the lack of tolerance to the Ig-like domain extends also to the transmembrane and cytoplasmic domains of MOG.

In addition, in both genotypes, the humoral immune response was mainly directed against the 1-25 and 50-79 amino acid sequences, as previously described in C57BL/6 mice (25). These functional studies demonstrate the lack of detectable B cell immunological tolerance toward the extracellular domain of MOG in the $\mathrm{MOG}^{+/+}$mice.

The lack of tolerance to MOG is corroborated by the fact that the MOG-specific $T$ cell repertoire of $\mathrm{MOG}^{+/+}$WT mice is equally encephalitogenic to that of $\mathrm{MOG}^{-/-}$mice, as shown in adoptive transfer experiments in which EAE is transferred to $\mathrm{MOG}^{+/+}$ recipients by either resting or primed $\mathrm{T}$ cells from $\mathrm{MOG}^{-/-}$and $\mathrm{MOG}^{+/+}$mice. Interestingly, these data are complementary to the findings in transgenic mice expressing MOG ubiquitously (under the control of MHC class I promoter), which are strongly resistant to both active and passive MOG EAE (A. Iglesias, unpublished data).

These findings imply that, at least in C57BL/6 mice, MOG is a myelin autoantigen to which no systemic immunological tolerance is induced during development or later in life, despite the presence of minute amounts of MOG transcripts in the thymus and spleen. By contrast, tolerance to other myelin proteins, such as MBP and DM20, can be readily detected $(4-6,28)$. This tolerance has been shown to be induced both in the thymus and in the periphery. MOG is, to our knowledge, the first direct demonstration of a self-antigen that does not promote immunological tolerance or substantially shape the self-repertoire. Triggering of this uncensored MOGspecific immune repertoire by priming of self-reactive lymphocytes in the periphery with either MOG or cross-reactive foreign antigens $(33,34)$ could well pose a potential threat to susceptible individuals. Such a state of immune ignorance to MOG can also be reversed during the course of inflammatory CNS diseases such as EAE and Theiler's virus encephalomyelitis, as a result of epitope spreading $(35,36)$. This is best exemplified in SJL/J mice infected with Theiler's virus, in which the earlier and stronger delayed-type hypersensitivity responses to myelin antigens involve the largely sequestered PLP 139-151 and MOG 92-106 peptides (36). Once elicited, this secondary anti-MOG immune response may contribute further to myelin destruction (35).

In other experimental systems, thymic expression of a supposedly organ-specific self-antigen correlated very closely with resistance to autoimmunity induced by the same self-antigen (37). This obviously applies only to individuals with MHC haplotypes that permit presentation of peptides derived from these self-antigens. In humans, a genetic polymorphism in the insulin-gene promoter, leading to reduced transcription of insulin in the thymus, contributes to genetic susceptibility to $\operatorname{IDDM}(38,39)$, while mutations in the AIRE (autoimmune regulator) gene, which encodes a transcription factor, lead to a monogenic multiorgan autoimmune disease, most likely because of a sharp decrease in promiscuous gene transcription in thymic epithelial cells (8). In myasthenia gravis, however, the major autoantigen is constitutively expressed in the thymus, but autoimmunity still develops (40). This could be related to differences in the thymic cell types presenting the self-antigen to developing thymocytes, the type of antigen-presenting cells being suggested to affect the efficiency of negative selection, and/or the generation of regulatory $\mathrm{T}$ cell populations (3). More generally, the cellular distribution and the expression level of autoantigens involved in organspecific autoimmunity should be thoroughly assessed in the thymus and secondary lymphoid organs of healthy as well as diseased individuals. This would permit the evaluation of the pathogenic or regulatory properties of autoantigen-specific $\mathrm{T}$ cells and thus aid the characterization of their roles in the pathophysiology of autoimmune disease.

In conclusion, this study using MOG knockout mice highlights the unique immunological features of MOG as compared with other self antigens. This model should also prove useful to analyze the role of MOG in myelin biology.

\section{Acknowledgments}

We thank Karine Goude, Sabine Desbois, and Sébastien Paturance for excellent technical assistance, and Hans Lassmann, Jean-Charles Guéry, Jean Kanellopoulos, and Christopher Linington for helpful discussions. We are indebted to André Calas, Université Pierre et Marie Curie, for his constant support. This work was supported by grants from the Association de Recherche sur la Sclérose en Plaques (to D. Pham-Dinh and R. Liblau), the European Leukodystrophy Association (to D. PhamDinh), and the European Union (no. QLRT-2001612 to J. Bauer and R. Liblau), and fellowships from the French Ministry of Education (to C. Delarasse), the Fondation pour la Recherche Médicale (to L.T. Mars), and the Netherlands Stichting Vrienden Multiple Sclerosis society (to S. Amor).

\footnotetext{
1. Ben-Nun, A., Wekerle, H., and Cohen, I.R. 1981. The rapid isolation of clonable antigen-specific $\mathrm{T}$ lymphocyte lines capable of mediating autoimmune encephalomyelitis. Eur. J. Immunol. 11:195-199.

2. Steinman, L. 1996. Multiple sclerosis: a coordinated immunological attack against myelin in the central nervous system. Cell. 85:299-302.

3. Sakaguchi, S. 2000. Regulatory T cells: key controllers of immunologic self-tolerance. Cell. 101:455-458.

4. Targoni, O.S., and Lehmann, P.V. 1998. Endogenous myelin basic protein inactivates the high avidity $\mathrm{T}$ cell repertoire. J. Exp. Med. 187:2055-2063.

5. Klein, L., Klugmann, M., Nave, K.A., Tuohy, V.K., and Kyewski, B. 2000. Shaping of the autoreactive T-cell repertoire by a splice variant of self protein expressed in thymic epithelial cells. Nat. Med. 6:56-61.
} 
6. Anderson, A.C., et al. 2000. High frequency of autoreactive myelin proteolipid protein-specific $T$ cells in the periphery of naive mice: mechanisms of selection of the self-reactive repertoire. J. Exp. Med. 191:761-770.

7. Derbinski, J., Schulte, A., Kyewski, B., and Klein, L. 2001. Promiscuous gene expression in medullary thymic epithelial cells mirrors the peripheral self. Nat. Immunol. 2:1032-1039.

8. Anderson, M.S., et al. 2002. Projection of an immunological self shadow within the thymus by the aire protein. Science. 298:1395-1401.

9. Linnington, C., Webb, M., and Woodhams, P.L. 1984. A novel myelinassociated glycoprotein defined by a mouse monoclonal antibody. J. Neuroimmunol. 6:387-396.

10. Lebar, R., Lubetzki, C., Vincent, C., Lombrail, P., and Boutry, J.M. 1986. The M2 autoantigen of central nervous system myelin, a glycoprotein present in oligodendrocyte membrane. Clin. Exp. Immunol. 66:423-434.

11. Pham-Dinh, D., et al. 1993. Myelin/oligodendrocyte glycoprotein is a member of a subset of the immunoglobulin superfamily encoded within the major histocompatibility complex. Proc. Natl. Acad. Sci. U. S. A. 90:7990-7994.

12. Amor, S., et al. 1994. Identification of epitopes of myelin oligodendrocyte glycoprotein for the induction of experimental allergic encephalomyelitis in $\mathrm{SJL}$ and Biozzi $\mathrm{AB} / \mathrm{H}$ mice. J. Immunol. 153:4349-4356.

13. Genain, C.P., et al. 1995. Antibody facilitation of multiple sclerosis-like lesions in a nonhuman primate. J. Clin. Invest. 96:2966-2974.

14. Mendel, I., Kerlero de Rosbo, N., and Ben-Nun, A. 1995. A myelin oligodendrocyte glycoprotein peptide induces typical chronic experimental autoimmune encephalomyelitis in $\mathrm{H}-2 \mathrm{~b}$ mice: fine specificity and $\mathrm{T}$ cell receptor $\mathrm{V}$ beta expression of encephalitogenic T cells. Eur. J. Immunol. 25:1951-1959.

15. Bernard, C.C., et al. 1997. Myelin oligodendrocyte glycoprotein: a novel candidate autoantigen in multiple sclerosis. J. Mol. Med. 75:77-88.

16. Iglesias, A., Bauer, J., Litzenburger, T., Schubart, A., and Linington, C. 2001. T- and B-cell responses to myelin oligodendrocyte glycoprotein in experimental autoimmune encephalomyelitis and multiple sclerosis. Glia. 36:220-234.

17. von Budingen, H.C., et al. 2001. Immune responses against the myelin/oligodendrocyte glycoprotein in experimental autoimmune demyelination. J. Clin. Immunol. 21:155-170.

18. Daubas, P., Pham-Dinh, D., and Dautigny, A. 1994. Structure and polymorphism of the mouse myelin/oligodendrocyte glycoprotein gene. Genomics. 23:36-41.

19. Le Mouellic, H., Lallemand, Y., and Brulet, P. 1990. Targeted replacement of the homeobox gene Hox-3.1 by the Escherichia coli lacZ in mouse chimeric embryos. Proc. Natl. Acad. Sci. U. S. A. 87:4712-4716.

20. della Gaspera, B., Pham-Dinh, D., Roussel, G., Nussbaum, J.L., and Dautigny, A. 1998. Membrane topology of the myelin/oligodendrocyte glycoprotein. Eur. J. Biochem. 258:478-484.

21. Norton, W.T., and Poduslo, S.E. 1973. Myelination in rat brain: method of myelin isolation. J. Neurochem. 21:749-757.

22. Liedtke, W., Edelmann, W., Chiu, F.C., Kucherlapati, R., and Raine, C.S. 1998. Experimental autoimmune encephalomyelitis in mice lacking glial fibrillary acidic protein is characterized by a more severe clinical course and an infiltrative central nervous system lesion. Am. J. Pathol. 152:251-259.

23. Chang, T.T., Jabs, C., Sobel, R.A., Kuchroo, V.K., and Sharpe, A.H. 1999. Studies in B7-deficient mice reveal a critical role for B7 costimulation in both induction and effector phases of experimental autoimmune encephalomyelitis. J. Exp. Med. 190:733-740.
24. Mars, L.T., et al. 2002. Cutting edge: V alpha 14-J alpha 281 NKT cells naturally regulate experimental autoimmune encephalomyelitis in nonobese diabetic mice. J. Immunol. 168:6007-6011.

25. Litzenburger, T., et al. 1998. B lymphocytes producing demyelinating autoantibodies: development and function in gene-targeted transgenic mice. J. Exp. Med. 188:169-180.

26. Loirat, D., Lemonnier, F.A., and Michel, M.L. 2000. Multiepitopic HLA-A*0201-restricted immune response against hepatitis B surface antigen after DNA-based immunization. J. Immunol. 165:4748-4755.

27. Baumann, N., and Pham-Dinh, D. 2001. Biology of oligodendrocyte and myelin in the mammalian central nervous system. Physiol. Rev. 81:871-927.

28. Harrington, C.J., et al. 1998. Differential tolerance is induced in T cells recognizing distinct epitopes of myelin basic protein. Immunity. 8:571-580.

29. Tuohy, V.K., Lu, Z., Sobel, R.A., Laursen, R.A., and Lees, M.B. 1989. Identification of an encephalitogenic determinant of myelin proteolipid protein for SJL mice. J. Immunol. 142:1523-1527.

30. Tompkins, S.M., et al. 2002. De novo central nervous system processing of myelin antigen is required for the initiation of experimental autoimmune encephalomyelitis. J. Immunol. 168:4173-4183.

31. Zhong, M.C., Cohen, L., Meshorer, A., Kerlero de Rosbo, N., and BenNun, A. 2000. T-cells specific for soluble recombinant oligodendrocyte-specific protein induce severe clinical experimental autoimmune encephalomyelitis in $\mathrm{H}-2(\mathrm{~b})$ and $\mathrm{H}-2(\mathrm{~s})$ mice. J. Neuroimmunol. 105:39-45

32. Flugel, A., et al. 2001. Migratory activity and functional changes of green fluorescent effector cells before and during experimental autoimmune encephalomyelitis. Immunity. 14:547-560.

33. Mokhtarian, F., Zhang, Z., Shi, Y., Gonzales, E., and Sobel, R.A. 1999. Molecular mimicry between a viral peptide and a myelin oligodendrocyte glycoprotein peptide induces autoimmune demyelinating disease in mice. J. Neuroimmunol. 95:43-54.

34. Stefferl, A., et al. 2000. Butyrophilin, a milk protein, modulates the encephalitogenic $\mathrm{T}$ cell response to myelin oligodendrocyte glycoprotein in experimental autoimmune encephalomyelitis. J. Immunol. 165:2859-2865.

35. McFarland, H.I., et al. 1999. Determinant spreading associated with demyelination in a nonhuman primate model of multiple sclerosis. J. Immunol. 162:2384-2390.

36. Katz-Levy, Y., et al. 2000. Temporal development of autoreactive Th1 responses and endogenous presentation of self myelin epitopes by central nervous system-resident APCs in Theiler's virus-infected mice. J. Immunol. 165:5304-5314.

37. Egwuagu, C.E., Charukamnoetkanok, P., and Gery, I. 1997. Thymic expression of autoantigens correlates with resistance to autoimmune disease. J. Immunol. 159:3109-3112.

38. Pugliese, A., et al. 1997. The insulin gene is transcribed in the human thymus and transcription levels correlated with allelic variation at the INS VNTR-IDDM2 susceptibility locus for type 1 diabetes. Nat. Genet. 15:293-297.

39. Vafiadis, P., et al. 1997. Insulin expression in human thymus is modulated by INS VNTR alleles at the IDDM2 locus. Nat. Genet. 15:289-292.

40. Salmon, A.M., Bruand, C., Cardona, A., Changeux, J.P., and BerrihAknin, S. 1998. An acetylcholine receptor alpha subunit promoter confers intrathymic expression in transgenic mice. Implications for tolerance of a transgenic self-antigen and for autoreactivity in myasthenia gravis. J. Clin. Invest. 101:2340-2350. 